\title{
Effect of withholding early parenteral nutrition in PICU on ketogenesis as potential mediator of its outcome benefit
}

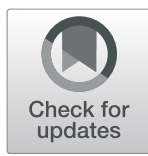

Astrid De Bruyn ${ }^{1+} \mathbb{D}$, Jan Gunst ${ }^{1+} \mathbb{D}$, Chloë Goossens ${ }^{1} \mathbb{D}$, Sarah Vander Perre', Gonzalo G. Guerra², Sascha Verbruggen ${ }^{3}$, Koen Joosten $^{3}$, Lies Langouche ${ }^{1+}$ (D) and Greet Van den Berghe ${ }^{1 * \dagger}$ (D)

\begin{abstract}
Background: In critically ill children, omitting early use of parenteral nutrition (late-PN versus early-PN) reduced infections, accelerated weaning from mechanical ventilation, and shortened PICU stay. We hypothesized that fasting-induced ketogenesis mediates these benefits.
\end{abstract}

Methods: In a secondary analysis of the PEPaNIC RCT ( $N=1440)$, the impact of late-PN versus early-PN on plasma 3-hydroxybutyrate (3HB), and on blood glucose, plasma insulin, and glucagon as key ketogenesis regulators, was determined for 96 matched patients staying $\geq 5$ days in PICU, and the day of maximal 3HB-effect, if any, was identified. Subsequently, in the total study population, plasma $3 \mathrm{HB}$ and late-PN-affected ketogenesis regulators were measured on that average day of maximal 3HB effect. Multivariable Cox proportional hazard and logistic regression analyses were performed adjusting for randomization and baseline risk factors. Whether any potential mediator role for $3 \mathrm{HB}$ was direct or indirect was assessed by further adjusting for ketogenesis regulators.

Results: In the matched cohort $(n=96)$, late-PN versus early-PN increased plasma 3HB throughout PICU days $1-5$ $(P<0.0001)$, maximally on PICU day 2. Also, blood glucose $(P<0.001)$ and plasma insulin $(P<0.0001)$, but not glucagon, were affected. In the total cohort ( $n=1142$ with available plasma), late-PN increased plasma $3 \mathrm{HB}$ on PICU day 2 (day 1 for shorter stayers) from (median [IQR]) 0.04 [0.04-0.04] mmol/L to 0.75 [0.04-2.03] mmol/L $(P<$ $0.0001)$. The $3 \mathrm{HB}$ effect of late-PN statistically explained its impact on weaning from mechanical ventilation ( $P=$ 0.0002) and on time to live PICU discharge $(P=0.004)$. Further adjustment for regulators of ketogenesis did not alter these findings.

Conclusion: Withholding early-PN in critically ill children significantly increased plasma 3HB, a direct effect that statistically mediated an important part of its outcome benefit.

Keywords: Parenteral nutrition, Intensive care, Ketogenesis, Ketone body, Recovery

\footnotetext{
* Correspondence: greet.vandenberghe@kuleuven.be

${ }^{+}$Astrid De Bruyn, Jan Gunst, Lies Langouche and Greet Van den Berghe contributed equally to this work.

${ }^{1}$ Clinical Division and Laboratory of Intensive Care Medicine, Department of Cellular and Molecular Medicine, KU Leuven, 3000 Leuven, Belgium

Full list of author information is available at the end of the article
} 


\section{Introduction}

Critically ill patients treated in the pediatric intensive care unit (PICU) often develop a pronounced macronutrient deficit because of the inability to feed orally and because nutrition administered via nasogastric tubes is poorly tolerated. The degree of the accumulated macronutrient deficit has been associated with poor outcome and delayed recovery $[1,2]$. However, this association is confounded by illness severity, given that the sicker patients are the ones to poorly tolerate enteral feeding. Furthermore, recent randomized controlled trials (RCTs) with variable design have not shown unequivocal benefit by early supplementation of insufficient or failing enteral nutrition, whereby some RCTs even indicated potential harm [3-7]. Indeed, the Early versus Late Parenteral Nutrition in Critically Ill Adults (EPaNIC) and Paediatric Early versus Late Parenteral Nutrition In Critical Illness (PEPaNIC) RCTs have shown that withholding parenteral nutrition until beyond the first week in intensive care (late-PN) was clinically superior to early parenteral nutrition supplementing insufficient enteral nutrition (early-PN) [4, 5]. As compared with early-PN, late-PN shortened dependency on intensive medical care in both RCTs, with a shorter duration of mechanical ventilatory support, fewer newly acquired infections, and a shorter intensive care and hospital stay $[4,5]$. In critically ill adults, late-PN also was found to lower the incidence of weakness [8]. Nevertheless, the optimal timing of initiating $\mathrm{PN}$ remains unclear.

One potential mediator of these clinical benefits brought about by accepting a macronutrient deficit early during critical illness is induction of a ketogenic fasting response. In healthy individuals, a sustained macronutrient deficit induces a fasting response with activated lipolysis and increased ketogenesis, together resulting in increased formation of the ketone bodies 3hydroxybutyrate (3HB) and acetoacetate [9]. Apart from being a vital, energy-efficient alternative fuel for the brain, heart, and skeletal muscle in times of fasting, ketone bodies also play an important signaling role. Ketone bodies enhance autophagy-driven cellular housekeeping and activate muscle regeneration $[10,11]$, pathways that have been shown to be hampered or insufficiently activated during critical illness [12, 13]. In addition, ketone bodies have anti-inflammatory properties [14]. Ketone bodies or ketogenic diets have shown to increase endurance in healthy athletes [15] and to induce beneficial effects in animal models of brain injury [16]. Moreover, in a mouse model of sepsis, a ketogenic PN formula and supplementation of $\mathrm{PN}$ with $3 \mathrm{HB}$ have shown to protect against the development of muscle weakness [17].

It remains unknown, however, whether critically ill patients can increase ketogenesis in response to the illnessassociated macronutrient deficit, and whether such ketogenic response-if present-is beneficial for recovery. In healthy subjects, fasting-induced ketogenesis is mediated by low circulating levels of insulin and glucose as well as high levels of glucagon, cortisol, and catecholamines [18-20]. In critical illness, however, although plasma glucagon, cortisol, and catecholamines are elevated, concomitant hyperinsulinemia and hyperglycemia may suppress ketogenesis [20, 21]. In line with this, available data, though scarce, suggested suppressed ketogenesis during critical illness [22-25]. Withholding early-PN has shown to reduce the degree of hyperglycemia, to lower the insulin requirements to prevent hyperglycemia $[4,5]$, and to lower the insulin/glucagon ratio [26].

We hypothesized that withholding early-PN enhances ketogenesis during critical illness and that such fastinginduced ketogenesis could be a mediator of the previously demonstrated recovery-enhancing effect of omitting PN early during critical illness [5]. We tested these hypotheses in a secondary analysis of the PEPaNIC RCT.

\section{Methods \\ Patients and study design}

This is a secondary analysis of the multicenter (Leuven, $\mathrm{BE}$, Rotterdam, NL, Edmonton, CA) PEPaNIC randomized controlled trial (ClinicalTrials.gov NCT01536275, $n=1440$ ). Written informed consent was obtained from the parents or legal guardians. The institutional or national ethical review boards of the participating centers approved the study protocol which was performed in accordance with the 1964 Declaration of Helsinki and later amendments. The detailed study protocol and primary results of the PEPaNIC study have been published [5, 27]. The study investigated the effect of omitting the use of PN to beyond the first week in the PICU (late-PN) as compared with the use of PN to complete insufficient or failing enteral nutrition from PICU admission onwards (early-PN). In both randomization groups, enteral nutrition was initiated as soon as possible, and gradually advanced up to target as tolerated. Beyond the first week, both groups received supplemental $\mathrm{PN}$ as long as patients were not fully enterally fed. To match the fluid intake of patients in the early-PN group, patients in the late-PN group received an isovolemic intravenous mixture of dextrose $5 \%$ and normal saline. To prevent refeeding syndrome, patients in both groups received intravenous trace elements, minerals, and vitamins, started on day 2 and continued until full enteral nutrition. Hyperglycemia was prevented with the use of intravenous insulin infusion, with center-specific target ranges $[5,27]$.

First, to evaluate whether and in which timeframe late-PN versus early-PN would affect ketogenesis, a time course analysis was planned in a matched subset of 
patients (Fig. 1). All patients who stayed in the PICU for at least 5 days and for whom daily stored plasma samples were available from admission until day 5 in PICU were selected in the Leuven, Rotterdam, and Edmonton cohorts. For Edmonton, this resulted in 0 children; for Rotterdam, this selection resulted in a cohort of 45 children; for Leuven, this selection resulted in a cohort of 199 children. To avoid confounding of the centerspecific insulin treatment strategies which could have affected plasma $3 \mathrm{HB}$ concentrations differently, the Leuven cohort was further reduced to a similar size as the Rotterdam cohort $(n=51)$ by propensity score matching. The resulting total cohort consisted of $96 \mathrm{pa}-$ tients (47 early-PN and 49 late-PN patients) matched for demographics (age, gender, weight) and baseline type and severity of illness (nutritional risk level according to
STRONGkids category, probability of death score evaluated by PIM2 score, emergency versus planned admission, diagnostic group, need of hemodynamic assist device on admission, presence of infection on admission) (Table 1). In this set, the impact of late-PN versus early$\mathrm{PN}$ on daily plasma concentrations of $3 \mathrm{HB}$ as well as on blood glucose, plasma insulin, and glucagon concentrations, potential regulators of ketogenesis, was determined. The time point of the maximal 3HB effect, if any, was then identified. Second (Fig. 1), for all patients in the PEPaNIC trial who had a plasma sample available for that average day of maximal effect (or last day in PICU for patients who were discharged from or died in the PICU before that day), plasma $3 \mathrm{HB}$ concentrations and also blood glucose, plasma insulin, and glucagon concentrations-if found to be affected in the above time
Step 1. Time course analysis $(\mathrm{n}=96)$

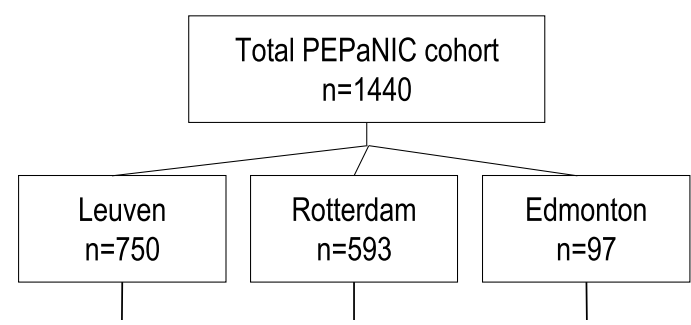

Patients with a PICU stay of $\geq 5$ days and available stored plasma samples from admission until day 5 in PICU
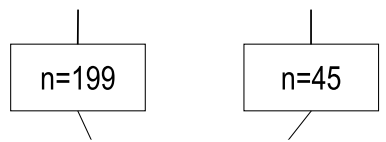

Matching for centre, demographics and type and severity of illness

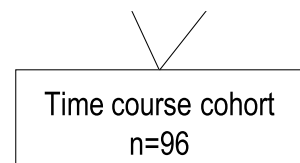

Quantification of daily plasma $3 H B$ and ketogenic regulators Identification of the time point of maximal $3 \mathrm{HB}$ effect
Step 2. Total study cohort $(n=1142)$

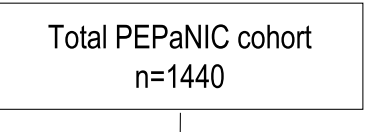

Patients with available stored plasma sample on day 2 (or day 1 for shorter stayers)

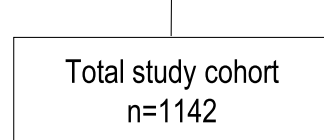

Quantification of plasma $3 H B$ concentration Quantification of affected ketogenic regulators

Step 3. Multivariable outcome analysis $(n=1142)$

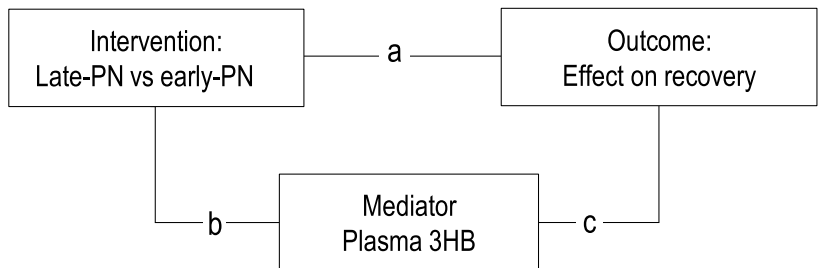

Fig. 1 Rationale and stepwise design of the study. In step 1, a time course analysis to investigate any impact of late-PN versus early-PN on daily plasma concentrations of 3HB. Therefore, all patients with a PICU stay of at least 5 days and plasma samples available from admission until day 5 in the PICU were selected and matched for center, demographics, and type and severity of illness. In the resulting matched cohort of 96 children, daily plasma $3 \mathrm{HB}$, insulin, glucagon, and glucose concentrations were determined and the time point of "maximal $3 \mathrm{HB}$ effect" was identified. In step 2, in the total study population, the concentration of plasma $3 \mathrm{HB}$ and in step 1 affected ketogenic regulators were determined on the average time point of "maximal 3HB effect" identified in step 1. For patients with a shorter PICU stay, the sample on the last day in PICU was used as surrogate (1142 patients with available plasma, of which 580 randomized to late-PN and 562 randomized to early-PN). In step 3, a multivariable statistical analysis was performed to assess whether any impact of late-PN as compared with early-PN on 3HB could explain its beneficial effects on outcome. "a" represents the effect of the intervention (late-PN versus early-PN) on the outcome of interest (time to live weaning from mechanical ventilatory support, time to live PICU discharge, and acquisition of new infection), "b" represents the effect of the intervention (late-PN versus early-PN) on the hypothesized mediator-plasma 3HB concentration, and " $\mathrm{C}$ " represents the association of the potential mediator with the outcome of interest (time to live weaning from mechanical ventilatory support, time to live PICU discharge, and acquisition of new infection) 
Table 1 Baseline characteristics of matched patients in the time course study

\begin{tabular}{|c|c|c|c|}
\hline Baseline characteristics & Early-PN $(n=47)$ & Late-PN $(n=49)$ & $P$ value \\
\hline$\overline{\text { Age (years)-median }[\mathrm{QQR}]^{2}}$ & $4.3[0.2-8.4]$ & $3.5[0.4-11.6]$ & 0.4 \\
\hline Age < 1 year-no. (\%) & $16(34.0)$ & $18(36.7)$ & 0.8 \\
\hline Male sex-no. (\%) & $28(59.5)$ & $34(69.3)$ & 0.3 \\
\hline Weight (kg) —-median $[\mathrm{IQR}]^{2}$ & $15.0[4.7-24.0]$ & $14.0[5.6-34.0]$ & 0.5 \\
\hline STRONGkids risk level—no. $(\%)^{1}$ & & & 1 \\
\hline Medium (1-3) & $44(93.6)$ & $46(93.8)$ & \\
\hline High $(4,5)$ & $3(6.3)$ & $3(6.1)$ & \\
\hline PIM2 calc. risk of death (\%)—-median $[\mathrm{IQR}]^{2}$ & $0.20[0.08-0.37]$ & $0.16[0.06-0.33]$ & 0.6 \\
\hline Emergency admission—no. (\%) & $16(34.0)$ & $15(30.6)$ & 0.8 \\
\hline \multicolumn{4}{|l|}{ Center-no. (\%) } \\
\hline Leuven & $25(53.1)$ & $26(53.0)$ & \\
\hline Rotterdam & $22(46.8)$ & $23(46.9)$ & \\
\hline Edmonton & $0(0)$ & $0(0)$ & \\
\hline \multicolumn{4}{|l|}{ Diagnostic group—no. (\%) } \\
\hline \multicolumn{4}{|l|}{ Surgical } \\
\hline Abdominal & $0(0)$ & $2(4.08)$ & \\
\hline Burns & $0(0)$ & $0(0)$ & \\
\hline Cardiac & $16(34.0)$ & $18(36.7)$ & \\
\hline Neurosurgery-traumatic brain injury & $8(17.0)$ & $7(14.2)$ & \\
\hline Thoracic & $0(0)$ & $0(0)$ & \\
\hline Transplantation & $4(8.5)$ & $2(4.1)$ & \\
\hline Orthopedic surgery-trauma & $3(6.3)$ & $0(0)$ & \\
\hline Others & $1(2.1)$ & $0(0)$ & \\
\hline \multicolumn{4}{|l|}{ Medical } \\
\hline Cardiac & $2(4.2)$ & $5(10.2)$ & \\
\hline Gastrointestinal-hepatic & $0(0)$ & $0(0)$ & \\
\hline Oncologic-hematologic & $0(0)$ & $1(2.0)$ & \\
\hline Neurologic & $1(2.1)$ & $3(6.1)$ & \\
\hline Respiratory & $5(10.6)$ & $7(14.2)$ & \\
\hline Others & $7(14.8)$ & $4(8.1)$ & \\
\hline \multicolumn{4}{|l|}{ Condition on admission—no. (\%) } \\
\hline Need for hemodynamic assist device & $1(0.02)$ & $1(0.02)$ & 0.2 \\
\hline Presence of infection & $26(55.3)$ & $24(48.9)$ & 0.5 \\
\hline
\end{tabular}

${ }^{1}$ Scores on the Screening Tool for Risk on Nutritional Status and Growth (STRONGkids) range from 0 to 5 , with a score of 0 indicating a low risk of malnutrition, a score of 1 to 3 indicating medium risk, and a score of 4 to 5 indicating high risk. ${ }^{2}$ Pediatric index of mortality 2 (PIM2) is a severity scoring system for predicting outcome of patients admitted to pediatric intensive care units

course study-were determined and compared for the late-PN and early-PN groups. Third (Fig. 1), a multivariate mediation analysis [28-30] was then performed on the total study cohort to assess whether any impact of late-PN as compared with early- $\mathrm{PN}$ on $3 \mathrm{HB}$ could explain its beneficial effects on outcome, adjusted for demographics, baseline risk factors, and type and severity of illness. If so, it was further assessed whether such a mediation role for $3 \mathrm{HB}$ was direct or indirect via an effect of late-PN on key regulators of ketogenesis, again adjusted for demographics, baseline risk factors, and type and severity of illness.

\section{Plasma analyses}

Plasma insulin and plasma glucagon concentrations were measured with commercial ELISAs (Invitrogen Ltd., Waltham, MA, USA, and Mercodia, Uppsala, Sweden), and blood glucose concentrations with the use of the ABL Radiometer. Plasma $3 \mathrm{HB}$ was quantified with a laboratory assay based upon the oxidation of $3 \mathrm{HB}$ to 
acetoacetate by the enzyme 3-hydroxybutyrate dehydrogenase and the concomitant reduction of cofactor $\mathrm{NAD}+$ to NADH [31]. Plasma samples were added to a reaction mixture containing EDTA ( $2 \mathrm{mM})$, sodium oxamate $(13 \mathrm{mM}), \mathrm{NAD}(3 \mathrm{mM})$, and 3-hydroxybutyrate dehydrogenase (1.5 U/ml, 3HBDB-RO Sigma-Aldrich) in Bis-Tris-Propane Buffer (50 mM, pH 9.5). The change in $\mathrm{NADH}$ fluorescence, compared to sample with reaction mixture without dehydrogenase, measured in a black 96well plate with transparent bottom after $15 \mathrm{~min}$ at $30^{\circ} \mathrm{C}$, is directly related to the $3 \mathrm{HB}$, with a detection limit of $0.04 \mathrm{mmol} / \mathrm{l}$ (excitation $340 \mathrm{~nm}$, emission $445 \mathrm{~nm}$, Tecan Infinite 200, Tecan Ltd., Männedorf, Switzerland).

\section{Statistical analyses}

Data are presented as frequencies and percentages or medians with interquartile range. Fisher's exact test and Kruskal-Wallis test were used to analyze univariable differences between patient groups, as appropriate. Multivariable Cox proportional hazard analysis was used to assess (first) whether randomization to late-PN or earlyPN independently associated with the time to live weaning from mechanical ventilatory support and the time to live discharge from the PICU, and (second) whether $3 \mathrm{HB}$ concentrations on the average day of maximal effect (or last day in PICU for patients with a shorter PICU stay) independently associated with these outcomes when added to this model. Likewise, multivariable logistic regression analysis was used to study whether randomization and $3 \mathrm{HB}$ concentrations independently associated with the incidence of a new infection in the PICU. Multivariable Cox proportional hazard and logistic regression analyses were adjusted for baseline risk factors (age, weight, gender, emergency versus elective admission, diagnostic group, PIM2 score, STRONGkids category, need of hemodynamic assist device on admission, presence of infection on admission), with censoring performed at 90 days or death. In those cases where adding the plasma $3 \mathrm{HB}$ concentration to the multivariable models showed that they were independently associated with the outcome of interest, hereby replacing the effect of the randomization to late- $\mathrm{PN}$ versus early- $\mathrm{PN}$ on the outcome, the $3 \mathrm{HB}$ effect was considered a statistical mediator of the late-PN outcome effect. To assess whether any potential mediator role for $3 \mathrm{HB}$ was direct or indirect via an effect of late-PN on key regulators of ketogenesis (plasma insulin, glucagon, and/or blood glucose concentrations) [18], sensitivity analyses were performed in which models were further adjusted for those regulators that were found to be affected by late-PN. In addition, multivariable logistic regression analysis was used to investigate the independent association between plasma $3 \mathrm{HB}$ concentration and PICU mortality. All analyses were performed with the use of JMP software, version pro 14 (SAS Institute, NC, USA). Statistical significance was set at a $P$ value of 0.0083 for the time course analysis (Bonferroni correction for multiple testing) and at 0.05 for other comparisons.

\section{Results}

Effect of late-PN versus early-PN on daily plasma concentrations of $3 \mathrm{HB}$ and identification of the time point of the maximal effect, if any

The matched cohort of patients with plasma samples available for each of the first 5 PICU days comprised 47 early-PN and 49 late-PN patients (Table 1, Fig. 1). Due to practical reasons, there was a slight time delay between initiation of the randomized intervention at PICU admission and the moment of drawing the admission sample, ranging from 0 to $7 \mathrm{~h}$ (median [IQR] 36 [18-74] $\mathrm{min})$.

Compliant with the study protocol, the daily total caloric intake was lower in late-PN patients than in earlyPN patients throughout the 5 first days in PICU (all $P<$ 0.0001 ) (Fig. 2a). Similar as in the total study population, late-PN patients developed an important macronutrient deficit over the first week in the PICU as compared to early-PN patients. From day 1 to day 5, plasma insulin concentrations were lower in late-PN patients than in early-PN patients (all $P<0.0001$ ) (Fig. 2b). Plasma glucagon concentrations were not different (Fig. 2c). From admission until day 2, blood glucose concentrations were lower in the late-PN group (all $P \leq 0.004$ ) (Fig. 1d). Throughout the 5 days, plasma $3 \mathrm{HB}$ concentrations were higher in late-PN patients than in early-PN patients $(P=$ 0.02 for admission and $P<0.0001$ for days 1 to 5 ), with the largest difference observed for day 2 (Fig. 2e).

\section{Effect of late-PN versus early-PN on plasma 3HB concentration on the "maximal effect day" in the total study cohort}

Given that day 2 in PICU was identified as the day upon which the difference in median $3 \mathrm{HB}$ between late-PN patients and early-PN patients was the largest, plasma $3 \mathrm{HB}$ was quantified for all study patients with an available plasma sample on day $2(n=822)$. For patients with a PICU stay of less than 2 days, the plasma samples of day 1 were used $(n=320)$ as surrogate.

For these 1142 patients, demographics, admission diagnosis, and baseline severity of illness were comparable between the 580 late-PN patients and 562 early-PN patients (Table 2). Cumulative caloric intake until blood sampling was lower in late-PN patients than in early-PN patients $(P<0.0001)$ (Fig. 3a). As in the time course study, plasma insulin and blood glucose concentrations were also lower in late-PN than in early-PN patients (both $P<0.0001$ ) (Fig. 3b, c). Given that the time course study did not show an effect of late-PN on plasma 

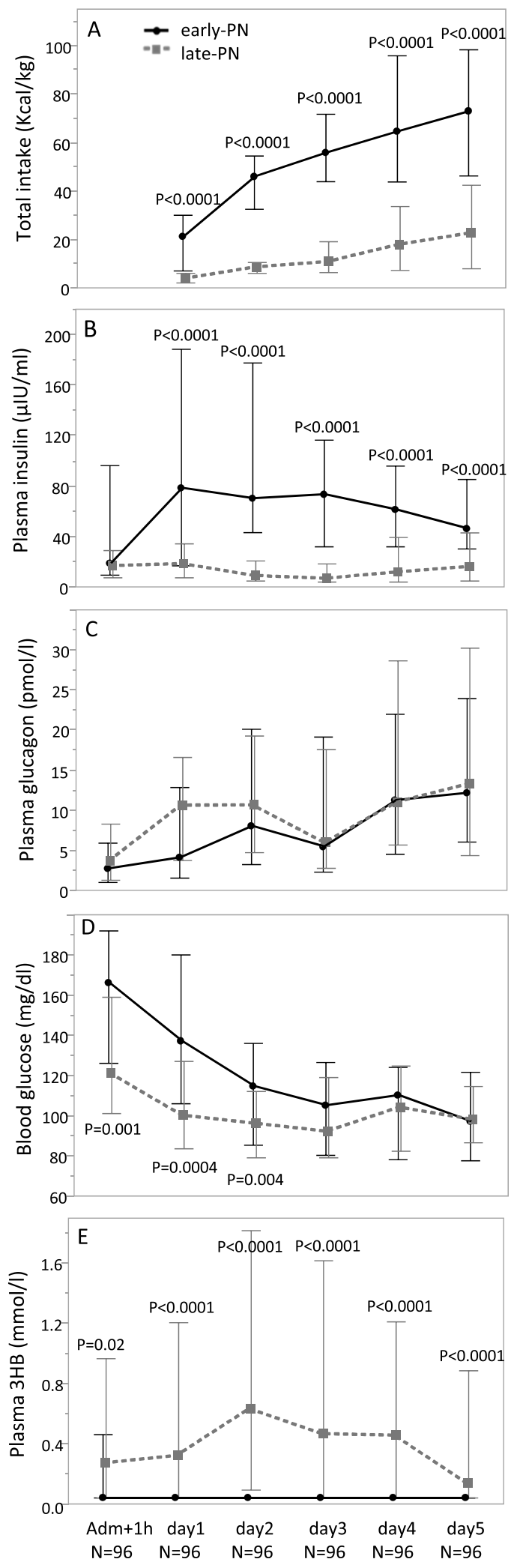

Fig. 2 Total caloric intake, plasma insulin, plasma glucagon, blood glucose, and plasma 3HB concentrations during the first 5 days in PICU in a matched cohort of early-PN and late-PN patients. Data are shown as median and IQR

glucagon, this analyte was not measured for the entire study cohort. Plasma $3 \mathrm{HB}$ concentrations were significantly higher in late-PN than in early-PN patients ((median [IQR]) $0.04 \quad[0.04-0.04] \quad \mathrm{mmol} / \mathrm{L}$ versus $0.75[0.04-2.03] \mathrm{mmol} / \mathrm{L} ; P<0.0001$ ) (Fig. 3d).

\section{Multivariable outcome analysis to assess whether the impact of late-PN versus early-PN on $3 \mathrm{HB}$ could explain its beneficial effects on outcome}

In the total study cohort $(n=1142)$, and adjusted for baseline risk factors, randomization to late-PN as compared with early-PN was independently associated with a higher likelihood of an earlier live weaning from mechanical ventilation and earlier live discharge from the PICU and with less newly acquired infections in the PICU (Table 3, panel 1). Adding the plasma 3HB concentrations measured on day 2 (or day 1 for shorter stayers) to the multivariable models revealed that a higher plasma $3 \mathrm{HB}$ concentration was independently associated with a higher likelihood of earlier live weaning from mechanical ventilatory support $(P=0.0002)$ and of earlier live PICU discharge $(P=0.004)$, hereby replacing the effect of randomization to late-PN versus early-PN (Table 3, panel 2), thus suggesting a mediator role for the $3 \mathrm{HB}$ effect of late-PN on these two outcomes. Further adjustment for the affected key regulators of ketogenesis (plasma insulin concentration, blood glucose concentration) did not alter these results (Table 3, panel 3 ). The effect of late-PN versus early-PN on plasma $3 \mathrm{HB}$ did not explain its impact on infections (Table 3, panels 2 and 3 ).

Plasma $3 \mathrm{HB}$ concentrations were not independently associated with PICU mortality (Additional Table 1).

\section{Discussion}

This secondary analysis of the PEPaNIC RCT revealed that late-PN, as compared with early-PN, associated with increased plasma $3 \mathrm{HB}$ concentrations from the first hours in PICU onward, sustained for at least 5 days, and with a maximal rise observed on PICU day 2. Also, levels of the key regulators of ketogenesis, blood glucose and insulin, were lowered whereas glucagonemia was unaffected. Statistical mediation analyses suggested that the rise in plasma $3 \mathrm{HB}$ concentrations with late- $\mathrm{PN}$, as compared with early-PN, explained its accelerating impact on live weaning from mechanical ventilatory support and on live discharge from the PICU, but not its preventive effect on newly acquired infections. This role of increased $3 \mathrm{HB}$ as a potential mediator of 2 major 
Table 2 Baseline characteristics of patients in the total study cohort

\begin{tabular}{|c|c|c|c|}
\hline Baseline characteristics & Early-PN $(n=580)$ & Late-PN $(n=562)$ & $P$ value \\
\hline$\overline{\text { Age (years)-median }[\mathrm{IQR}]^{2}}$ & $1.8[0.3-6.7]$ & $1.9[0.3-8.4]$ & 0.4 \\
\hline Age < 1 year-no. (\%) & $238(41.0)$ & $228(40.5)$ & 0.9 \\
\hline Male gender-no. (\%) & $323(55.6)$ & $324(57.6)$ & 0.5 \\
\hline Weight (kg)—median $[\mathrm{IQR}]^{2}$ & $11.1[5.3-21.0]$ & $12.0[5.1-25.0]$ & 0.3 \\
\hline STRONGkids risk level—no. (\%) ${ }^{1}$ & & & 0.8 \\
\hline Medium (1-3) & $530(91.3)$ & $511(90.9)$ & \\
\hline High $(4,5)$ & $50(8.6)$ & $51(9.0)$ & \\
\hline PIM2 calc. risk of death (\%)—-median $[\mathrm{IQR}]^{2}$ & $0.06[0.02-0.21]$ & $0.06[0.02-0.017]$ & 0.5 \\
\hline Emergency admission—no. (\%) & $272(46.9)$ & $271(48.2)$ & 0.6 \\
\hline \multicolumn{4}{|l|}{ Center-no. (\%) } \\
\hline Leuven & $371(63.9)$ & $372(66.1)$ & \\
\hline Rotterdam & $173(29.8)$ & $161(28.6)$ & \\
\hline Edmonton & $36(6.2)$ & $29(5.1)$ & \\
\hline \multicolumn{4}{|l|}{ Diagnostic group—no. (\%) } \\
\hline \multicolumn{4}{|l|}{ Surgical } \\
\hline Abdominal & $25(4.3)$ & $27(4.8)$ & \\
\hline Burns & $3(0.5)$ & $5(0.8)$ & \\
\hline Cardiac & $266(45.8)$ & $255(45.3)$ & \\
\hline Neurosurgery-traumatic brain injury & $59(10.1)$ & $46(8.1)$ & \\
\hline Thoracic & $24(4.1)$ & $20(3.5)$ & \\
\hline Transplantation & $7(1.2)$ & $15(2.6)$ & \\
\hline Orthopedic surgery-trauma & $27(4.6)$ & $26(4.6)$ & \\
\hline Others & $11(1.9)$ & $19(3.3)$ & \\
\hline \multicolumn{4}{|l|}{ Medical } \\
\hline Cardiac & $22(3.7)$ & $22(3.9)$ & \\
\hline Gastrointestinal-hepatic & $2(0.3)$ & $3(0.5)$ & \\
\hline Oncologic-hematologic & $4(0.6)$ & $7(1.2)$ & \\
\hline Neurologic & $41(7.0)$ & $36(6.4)$ & \\
\hline Respiratory & $54(9.3)$ & $52(9.2)$ & \\
\hline Others & $35(6.0)$ & $29(5.1)$ & \\
\hline \multicolumn{4}{|l|}{ Condition on admission—no. (\%) } \\
\hline Need for hemodynamic assist device & $17(0.0)$ & $23(0.0)$ & 0.2 \\
\hline Presence of infection & $212(36.5)$ & $193(34.3)$ & 0.4 \\
\hline
\end{tabular}

${ }^{1}$ Scores on the Screening Tool for Risk on Nutritional Status and Growth (STRONGkids) range from 0 to 5, with a score of 0 indicating a low risk of malnutrition, a score of 1 to 3 indicating medium risk, and a score of 4 to 5 indicating high risk. ${ }^{2}$ Pediatric index of mortality 2 (PIM2) is a severity scoring system for predicting outcome of patients admitted to pediatric intensive care units

outcome benefits of late-PN was a direct one, as it was independent of the observed changes in the key regulators of ketogenesis.

With late-PN, and thus with accepting an important macronutrient deficit during the first week in the PICU, plasma $3 \mathrm{HB}$ concentrations were found to rise up to the millimolar range. This high level of plasma $3 \mathrm{HB}$ in response to late-PN was striking given that earlier studies had suggested impaired ketogenesis during critical illness in adults [22-25]. Hyperinsulinemia and hyperglycemia are powerful suppressors of ketogenesis and hallmarks of critical illness [20, 21]. Glucagon, cortisol, and catecholamines are hormones known to enhance ketogenesis in healthy subjects, and levels of these stress hormones are elevated during critical illness [18, 32]. However, as shown previously for cortisol [33], and now also for glucagon, not using early-PN did not affect the plasma levels of these ketogenic hormones in critically ill children. Also, the use of corticosteroids and of catecholamines was unaffected by late- $\mathrm{PN}$, as shown 


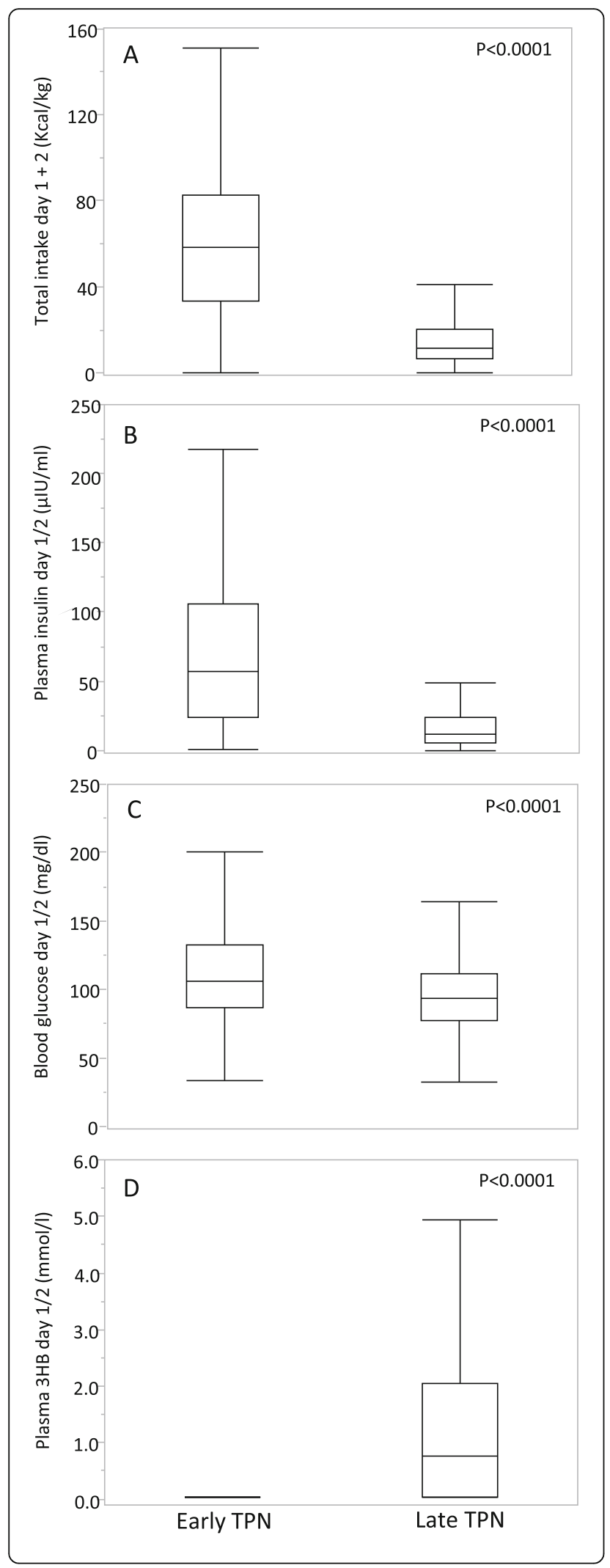

Fig. 3 Total caloric intake, plasma insulin, blood glucose, and plasma $3 \mathrm{HB}$ concentrations on day $1 / 2$ in the total cohort of early-PN and late-PN patients. Plasma concentrations of 3-HB and insulin were determined on day 2 in PICU or on day 1 for patients with a shorter PICU stay. Boxes show median and IQR, whiskers 10th and 90th percentile

previously [5, 33]. In contrast, not using early-PN lowered blood glucose and plasma insulin concentrations in the current study, in line with the earlier finding of a lowered insulin requirement to prevent hyperglycemia $[4,5]$. Together, this suggests that late-PN, as compared with early-PN, increased plasma $3 \mathrm{HB}$ concentrations throughout the first 5 days in the PICU by reducing suppressors of ketogenesis rather than by enhancing ketogenesis activators.

With late-PN, the highest rise in plasma $3 \mathrm{HB}$ concentrations was observed on day 2 in the PICU with concentrations declining somewhat thereafter, though staying elevated throughout the first 5 days in the PICU. This decline corresponds with the increase in the amount of enteral nutrition from day 2 onwards, although that amount was still small. It is well known that ketogenesis is very sensitive to suppression by macronutrient intake [9]. Also, given that the blood glucose levels were no longer different after PICU day 2, and hyperglycemia is a known suppressor of ketogenesis [22], the blood glucose effect may have contributed to the peak rise in plasma $3 \mathrm{HB}$ on that day. Remarkably, although children were not fully fasted during these first PICU days, receiving minimal doses of IV glucose and receiving blood glucose control with insulin infusion, the plasma ketone concentrations in the late-PN group rose quite high, up to the millimolar range. This may have to do with the young age of the critically ill patients of this study, as the ketogenic fasting response in children is known to be more pronounced than in adults [9, 22-25].

The statistical mediation analyses suggested that the rise in plasma $3 \mathrm{HB}$ concentrations with late- $\mathrm{PN}$, as compared with early-PN, explained its accelerating impact on live weaning from mechanical ventilatory support and on live discharge from the PICU, but not its preventive effect on newly acquired infections. The mechanism by which $3 \mathrm{HB}$ may enhance recovery from critical illness cannot be identified in the current study. One possibility is that $3 \mathrm{HB}$, during critical illness, similarly as during prolonged fasting in healthy subjects, serves as a vital or super fuel for the brain, heart, and skeletal muscle [9]. In healthy athletes, ingestion of $3 \mathrm{HB}$ esters has shown to increase endurance [15]. Also, in a mouse model of sepsis, a ketogenic PN formula and supplementation of $\mathrm{PN}$ with $3 \mathrm{HB}$ have shown to protect against 
Table 3 A 3-step approach to investigate a potential mediation role of the effect on 3HB for the outcome benefits of late-PN

\begin{tabular}{|c|c|c|c|c|c|c|}
\hline \multirow[b]{2}{*}{ A. Impact of randomization $(n=1142)$} & \multicolumn{2}{|c|}{$\begin{array}{l}\text { Likelihood of live weaning } \\
\text { from mechanical ventilatory } \\
\text { support }\end{array}$} & \multicolumn{2}{|c|}{$\begin{array}{l}\text { Likelihood of live discharge } \\
\text { from the PICU }\end{array}$} & \multicolumn{2}{|c|}{$\begin{array}{l}\text { Acquisition of a new infection } \\
\text { in the PICU }\end{array}$} \\
\hline & Risk ratio $(95 \% \mathrm{Cl})$ & $P$ value & Risk ratio $(95 \% \mathrm{Cl})$ & $P$ value & Odds ratio $(95 \% \mathrm{Cl})$ & $P$ value \\
\hline $\begin{array}{l}\text { Randomization to late-PN ( } n=562) \\
\text { vs. early-PN }(n=580)\end{array}$ & $1.14(1.01-1.29)$ & 0.02 & $1.19(1.06-1.35)$ & 0.003 & $0.49(0.34-0.70)$ & $<0.0001$ \\
\hline \multicolumn{7}{|l|}{ Demographics } \\
\hline Age per year added & $1.02(0.98-1.05)$ & 0.18 & $1.02(0.99-1.06)$ & 0.11 & $1.02(0.93-1.13)$ & 0.59 \\
\hline Weight per kg added & $0.99(0.98-1.00)$ & 0.65 & $0.99(0.98-1.00)$ & 0.34 & $0.99(0.96-1.02)$ & 0.72 \\
\hline Male gender & $0.98(0.85-1.11)$ & 0.85 & $0.94(0.83-1.07)$ & 0.39 & $1.11(0.78-1.58)$ & 0.52 \\
\hline \multicolumn{7}{|l|}{ Characteristics of type and severity of illness } \\
\hline Emergency vs. planned admission & $0.74(0.61-0.90)$ & 0.003 & $0.77(0.63-0.93)$ & 0.008 & $1.74(1.03-2.92)$ & 0.03 \\
\hline Diagnostic group & & 0.22 & & 0.40 & & 0.06 \\
\hline PIM2 score per point added & $0.70(0.66-0.74)$ & $<0.0001$ & $0.72(0.68-0.76)$ & $<0.0001$ & $1.41(1.25-1.60)$ & $<0.0001$ \\
\hline $\begin{array}{l}\text { High vs. medium risk of malnutrition } \\
\text { (STRONGkids risk level } 4-5 \text { vs. 1-3) }\end{array}$ & $0.86(0.68-1.06)$ & 0.17 & $0.83(0.67-1.03)$ & 0.1 & $1.15(0.65-2.02)$ & 0.62 \\
\hline Presence of infection on admission & $0.72(0.61-0.84)$ & $<0.0001$ & $0.71(0.61-0.84)$ & $<0.0001$ & $0.52(0.32-0.83)$ & 0.005 \\
\hline Need for hemodynamic assist device on admission & $0.51(0.34-0.76)$ & 0.001 & $0.48(0.32-0.73)$ & 0.0005 & $1.71(0.80-3.64)$ & 0.16 \\
\hline B. Mediation role for plasma 3HB $(n=1142)$ & Risk ratio $(95 \% \mathrm{Cl})$ & $P$ value & Risk ratio $(95 \% \mathrm{Cl})$ & $P$ value & Odds ratio $(95 \% \mathrm{Cl})$ & $P$ value \\
\hline Plasma 3 HB on day 2 (per $\mathrm{mmol} / \mathrm{l}^{1}$ & $1.12(1.05-1.20)$ & 0.0002 & $1.10(1.03-1.17)$ & 0.004 & $0.85(0.68-1.06)$ & 0.14 \\
\hline $\begin{array}{l}\text { Randomization to late-PN ( } n=562) \\
\text { vs. early-PN }(n=580)\end{array}$ & $1.01(0.88-1.16)$ & 0.79 & $1.08(0.94-1.24)$ & 0.26 & $0.56(0.38-0.84)$ & 0.004 \\
\hline \multicolumn{7}{|l|}{ Demographics } \\
\hline Age per year added & $1.02(0.99-1.06)$ & 0.11 & $1.03(0.99-1.06)$ & 0.06 & $1.02(0.92-1.12)$ & 0.64 \\
\hline Weight per kg added & $0.99(0.98-1.00)$ & 0.55 & $0.99(0.98-1.00)$ & 0.28 & $0.99(0.96-1.02)$ & 0.72 \\
\hline Male gender & $0.99(0.88-1.12)$ & 0.95 & $0.95(0.84-1.07)$ & 0.44 & $1.11(0.78-1.57)$ & 0.55 \\
\hline \multicolumn{7}{|l|}{ Characteristics of type and severity of illness } \\
\hline Emergency vs. planned admission & $0.77(0.63-0.94)$ & 0.01 & $0.79(0.65-0.96)$ & 0.02 & $1.65(0.98-2.79)$ & 0.05 \\
\hline Diagnostic group & & 0.27 & & 0.32 & & 0.07 \\
\hline PIM2 score per point added & $0.70(0.66-0.74)$ & $<0.0001$ & $0.72(0.68-0.76)$ & $<0.0001$ & $1.41(1.24-1.60)$ & $<0.0001$ \\
\hline High vs. medium risk of malnutrition & $0.85(0.68-1.05)$ & 0.14 & $0.83(0.67-1.03)$ & 0.09 & $1.16(0.66-2.05)$ & 0.59 \\
\hline Presence of infection on admission & $0.72(0.61-0.85)$ & $<0.0001$ & $0.72(0.61-0.84)$ & $<0.0001$ & $0.51(0.32-0.82)$ & 0.004 \\
\hline Need for hemodynamic assist device on admission & $0.50(0.33-0.76)$ & 0.001 & $0.48(0.32-0.73)$ & 0.0001 & $1.66(0.78-3.55)$ & 0.19 \\
\hline $\begin{array}{l}\text { C. Sensitivity analysis (direct/indirect 3HB effect) } \\
(n=1142)\end{array}$ & Risk ratio $(95 \% \mathrm{Cl})$ & $P$ value & Risk ratio $(95 \% \mathrm{Cl})$ & $P$ value & Odds ratio $(95 \% \mathrm{Cl})$ & $P$ value \\
\hline Plasma $3 \mathrm{HB}$ on day $2\left(\right.$ per $\mathrm{mmol} / \mathrm{l}^{1}$ & $1.12(1.05-1.19)$ & 0.0005 & $1.09(1.02-1.17)$ & 0.007 & $0.80(0.63-1.02)$ & 0.06 \\
\hline $\begin{array}{l}\text { Randomization to late-PN ( } n=562) \\
\text { vs. early-PN }(n=580)\end{array}$ & $0.97(0.83-1.12)$ & 0.70 & $1.04(0.89-1.21)$ & 0.60 & $0.62(0.40-0.94)$ & 0.02 \\
\hline \multicolumn{7}{|l|}{ Demographics } \\
\hline Age per year added & $1.03(0.99-1.06)$ & 0.08 & $1.03(0.99-1.06)$ & 0.06 & $1.03(0.93-1.14)$ & 0.50 \\
\hline Weight per kg added & $0.99(0.98-1.00)$ & 0.67 & $0.99(0.98-1.00)$ & 0.36 & $0.99(0.96-1.02)$ & 0.54 \\
\hline Male gender & $0.97(0.86-1.11)$ & 0.74 & $0.94(0.83-1.06)$ & 0.34 & $1.19(0.83-1.72)$ & 0.33 \\
\hline \multicolumn{7}{|l|}{ Characteristics of type and severity of illness } \\
\hline Emergency vs. planned admission & $0.72(0.59-0.89)$ & 0.002 & $0.76(0.62-0.93)$ & 0.01 & $1.62(0.94-2.79)$ & 0.07 \\
\hline Diagnostic group & & 0.28 & & 0.42 & & 0.03 \\
\hline PIM2 score per point added & $0.69(0.65-0.73)$ & $<0.0001$ & $0.72(0.68-0.75)$ & $<0.0001$ & $1.45(1.27-1.65)$ & $<0.0001$ \\
\hline High vs. medium risk of malnutrition & $0.84(0.67-1.04)$ & 0.12 & $0.82(0.65-1.02)$ & 0.07 & $1.15(0.64-2.06)$ & 0.63 \\
\hline Presence of infection on admission & $0.72(0.61-0.85)$ & 0.0001 & $0.72(0.62-0.85)$ & 0.0001 & $0.49(0.30-0.81)$ & 0.004 \\
\hline
\end{tabular}


Table 3 A 3-step approach to investigate a potential mediation role of the effect on 3HB for the outcome benefits of late-PN (Continued)

\begin{tabular}{|c|c|c|c|c|c|c|}
\hline \multirow[b]{2}{*}{$\begin{array}{l}\text { Need for hemodynamic assist device on } \\
\text { admission }\end{array}$} & \multicolumn{2}{|c|}{$\begin{array}{l}\text { Likelihood of live weaning } \\
\text { from mechanical ventilatory } \\
\text { support }\end{array}$} & \multicolumn{2}{|c|}{$\begin{array}{l}\text { Likelihood of live discharge } \\
\text { from the PICU }\end{array}$} & \multicolumn{2}{|c|}{$\begin{array}{l}\text { Acquisition of a new infection } \\
\text { in the PICU }\end{array}$} \\
\hline & $0.51(0.34-0.78)$ & 0.002 & $0.48(0.32-0.74)$ & 0.0008 & $1.44(0.66-3.13)$ & 0.36 \\
\hline \multicolumn{7}{|l|}{ Late-PN-affected regulators of ketogenesis } \\
\hline $\begin{array}{l}\text { Plasma insulin at time of } 3 \mathrm{HB} \text { assessment } \\
(\text { per } \mu \mathrm{lU} / \mathrm{l})\end{array}$ & $1.00(0.99-1.00)$ & 0.93 & $0.99(0.99-1.00)$ & 0.52 & $0.80(0.63-1.02)$ & 0.76 \\
\hline $\begin{array}{l}\text { Blood glucose at time of } 3 \mathrm{HB} \text { assessment } \\
\text { (per } \mathrm{mg} / \mathrm{dl} \text { ) }\end{array}$ & $0.99(0.99-0.99)$ & 0.02 & $0.99(0.99-1.00)$ & 0.46 & $0.99(0.99-1.00)$ & 0.49 \\
\hline
\end{tabular}

${ }^{1}$ Plasma 3 HB was quantified for all study patients with an available plasma sample on day $2(n=822)$ or day 1 ( $\left.n=320\right)$ for patients with a shorter PICU stay

the development of muscle weakness [17]. Alternatively, $3 \mathrm{HB}$ may act through enhanced autophagy-driven cellular housekeeping [10], a pathway that was previously shown to be activated by late-PN in critically ill adults [13]. Another possibility is a 3HB-driven activation of muscle regeneration, as was shown earlier in a mouse model of sepsis [11]. Finally, although $3 \mathrm{HB}$ may also have anti-inflammatory effects [14], such a mechanism appears less likely, given that late-PN has shown to increase plasma C-reactive protein concentrations, considered to be a marker of inflammation $[4,5]$. The finding that the rise in $3 \mathrm{HB}$ with late-PN did not explain its preventive impact on new infections also suggests that an immune modulating effect of $3 \mathrm{HB}$ does not seem to be involved.

Adjustment for key suppressors of ketogenesis, blood glucose and plasma insulin $[18,20,22]$, that were lowered by late-PN, did not affect the statistical mediating role of the rise in plasma $3 \mathrm{HB}$ concentrations with late$\mathrm{PN}$ on outcome. This suggests that the effect of the rise in $3 \mathrm{HB}$ on accelerated recovery was a direct one, rather than that it merely mirrored the impact of lowered insulin or blood glucose. This was further supported by the finding that plasma insulin concentrations were not independently associated with the late-PN-affected outcomes in the multivariable models. Interestingly, a lower blood glucose concentration was independently associated with a higher likelihood of earlier live weaning from mechanical ventilator support. Targeting normal fasting levels of blood glucose with insulin in adults has previously shown to protect against critical illness-induced polyneuropathy/myopathy, an important cause of limb and respiratory muscle weakness, and a complication that prolongs the need for mechanical ventilation [34, 35]. However, as compared with the large impact on blood glucose in those studies, the difference in blood glucose between late-PN and early-PN that was observed here was small. Altogether, our data open perspectives for studies investigating the impact of exogenous ketone supplementation or ketogenic diets on outcome of critically ill patients. Possibly, such strategies could allow effective and safe initiation of artificial feeding while avoiding prolonged fasting intervals, a hypothesis that requires further study. Evidently, future studies should also investigate potential side effects of such feeding strategies, such as gastrointestinal tolerance and, for ketogenic diets, metabolic acidosis.

One strength of this study is the design as a secondary analysis of a large RCT, with prospectively planned collection of plasma samples on a daily basis. This allowed to perform repeated measurements and to identify the time point of the maximal $3 \mathrm{HB}$ effect. This study has also limitations. First, not all patients of the original cohort had a sample available for the day of the maximal $3 \mathrm{HB}$ effect. However, samples were available for 1142 of the 1440 patients and the late-PN and early-PN groups were still comparable for demographics, admission diagnosis, and baseline severity of illness. Also, the outcome benefits of late-PN versus early-PN were present in the cohort of 1142 patients and comparable to those in the 1440 patient cohort. A second limitation is that the mediation analyses were performed with plasma $3 \mathrm{HB}$ concentrations quantified on a single time point, namely the day of the maximal $3 \mathrm{HB}$ effect. It thus remains unclear whether the observed positive effect of plasma $3 \mathrm{HB}$ concentrations on outcome is related to its peak effect or to the duration of enhanced ketogenesis during critical illness. Third, in the sensitivity mediation analyses, in which it was investigated whether the impact of late-PN versus early-PN on plasma insulin and blood glucose played a role, it cannot be excluded that other, yet unknown, factors were involved. Finally, this study was performed in a pediatric ICU population. Whether these findings can be extrapolated to adult ICU patients requires further investigation.

\section{Conclusion}

Withholding PN during the first week in the PICU, whereby suppressors of ketogenesis were reduced, rapidly increased plasma $3 \mathrm{HB}$ concentrations up to the 
millimolar range, an effect that was sustained for at least 5 days. This $3 \mathrm{HB}$ effect of withholding $\mathrm{PN}$ was found to be a direct statistical mediator of an important part of its beneficial impact on recovery. These findings open perspectives for the further investigation of ketone supplementation and of ketogenic diets to improve outcome of critically ill patients.

\section{Supplementary information}

Supplementary information accompanies this paper at https://doi.org/10. 1186/s13054-020-03256-z.

Additional file 1: Table 1. Association of plasma $3 \mathrm{HB}$ concentration with PICU mortality.

\section{Acknowledgements}

Not applicable

\section{Authors' contributions}

$A D, J G, L L$, and GVdB conceived and designed the experiments. SV, KJ, GGG, and $G V d B$ acquired the data. $A D, C G$, and SVP performed the laboratory experiments. $A D, J G, L L$, and $G V d B$ analyzed the data and wrote the manuscript. All authors read and approved the final manuscript.

\section{Funding}

This work was supported by ERC Advanced Grants from the Horizon 2020 Programme (AdvG-2017-785809) to GVdB; by the Methusalem programme of the Flemish Government (through the University of Leuven to GVdB and LL, METH14/06); by the Agency for Innovation through Science and Technology, Flanders, Belgium (through the University of Leuven to GVdB, IWT/070695/ TBM); by the Research Foundation Flanders (FWO), Belgium (to LL and GVdB, G.0C78.17N); by the University Hospitals Leuven (postdoctoral research fellowship by the Clinical Research and Education Council to JG); by the Sophia Children's Hospital Foundation (SSWO) to SCV; by the Stichting Agis Zorginnovatie to SCV; by the Erasmus Trustfonds to SCV; and by a European Society for Parenteral and Enteral Nutrition (ESPEN) research grant to SCV.

\section{Availability of data and materials}

Data sharing is offered under the format of collaborative projects. Proposals can be directed to the corresponding author.

\section{Ethics approval and consent to participate}

This is a secondary analysis of the multicenter (Leuven, BE, Rotterdam, NL, Edmonton, CA) PEPaNIC randomized controlled trial (ClinicalTrials.gov NCT01536275, $n=1440$ ). The institutional or national ethical review boards of the participating centers approved the study protocol. Written informed consent was obtained from the parents or legal guardians.

\section{Consent for publication}

Not applicable

\section{Competing interests}

We declare no competing interests.

\section{Author details}

${ }^{1}$ Clinical Division and Laboratory of Intensive Care Medicine, Department of Cellular and Molecular Medicine, KU Leuven, 3000 Leuven, Belgium. ${ }^{2}$ Department of Paediatrics, Intensive Care Unit, University of Alberta, Stollery Children's Hospital, Edmonton, AB, Canada. ${ }^{3}$ Intensive Care Unit, Department of Paediatrics and Paediatric Surgery, Erasmus Medical Centre-Sophia Children's Hospital, Rotterdam, Netherlands.
Received: 8 May 2020 Accepted: 17 August 2020

Published online: 31 August 2020

\section{References}

1. Alberda C, Gramlich L, Jones N, Jeejeebhoy K, Day AG, Dhaliwal R, et al. The relationship between nutritional intake and clinical outcomes in critically ill patients: results of an international multicenter observational study. Intensive Care Med. 2009;35(10):1728-37.

2. Joffe A, Anton $N$, Lequier L, Vandermeer B, Tjosvold L, Larsen B, et al. Nutritional support for critically ill children. Cochrane Database Syst Rev. 2016;(5):Cd005144. https://doi.org/10.1002/14651858.CD005144.pub3.

3. Doig GS, Simpson F, Sweetman EA, Finfer SR, Cooper DJ, Heighes PT, et al. Early parenteral nutrition in critically ill patients with short-term relative contraindications to early enteral nutrition: a randomized controlled trial. JAMA. 2013;309(20):2130-8.

4. Casaer MP, Mesotten D, Hermans G, Wouters PJ, Schetz M, Meyfroidt G, et al. Early versus late parenteral nutrition in critically ill adults. N Engl J Med. 2011;365(6):506-17.

5. Fivez T, Kerklaan D, Mesotten D, Verbruggen S, Wouters PJ, Vanhorebeek I, et al. Early versus late parenteral nutrition in critically ill children. N Engl J Med. 2016;374(12):1111-22.

6. Allingstrup MJ, Kondrup J, Wiis J, Claudius C, Pedersen UG, Hein-Rasmussen $\mathrm{R}$, et al. Early goal-directed nutrition versus standard of care in adult intensive care patients: the single-centre, randomised, outcome assessorblinded EAT-ICU trial. Intensive Care Med. 2017;43(11):1637-47.

7. Heidegger CP, Berger MM, Graf S, Zingg W, Darmon P, Costanza MC, et al. Optimisation of energy provision with supplemental parenteral nutrition in critically ill patients: a randomised controlled clinical trial. Lancet. 2013; 381(9864):385-93.

8. Hermans G, Casaer MP, Clerckx B, Guiza F, Vanhullebusch T, Derde S, et al. Effect of tolerating macronutrient deficit on the development of intensivecare unit acquired weakness: a subanalysis of the EPaNIC trial. Lancet Respir Med. 2013;1(8):621-9.

9. Cahill GF Jr. Fuel metabolism in starvation. Annu Rev Nutr. 2006;26:1-22.

10. Camberos-Luna L, Geronimo-Olvera C, Montiel T, Rincon-Heredia R, Massieu $\mathrm{L}$. The ketone body, beta-hydroxybutyrate stimulates the autophagic flux and prevents neuronal death induced by glucose deprivation in cortical cultured neurons. Neurochem Res. 2016;41(3):600-9.

11. Zou X, Meng J, Li L, Han W, Li C, Zhong R, et al. Acetoacetate accelerates muscle regeneration and ameliorates muscular dystrophy in mice. J Biol Chem. 2016;291(5):2181-95.

12. Puthucheary ZA, Rawal J, McPhail M, Connolly B, Ratnayake G, Chan P, et al. Acute skeletal muscle wasting in critical illness. JAMA. 2013; 310(15):1591-600

13. Vanhorebeek I, Gunst J, Derde S, Derese I, Boussemaere M, Guiza F, et al. Insufficient activation of autophagy allows cellular damage to accumulate in critically ill patients. J Clin Endocrinol Metab. 2011;96(4):E633-45.

14. Youm YH, Nguyen KY, Grant RW, Goldberg EL, Bodogai M, Kim D, et al. The ketone metabolite beta-hydroxybutyrate blocks NLRP3 inflammasomemediated inflammatory disease. Nat Med. 2015;21(3):263-9.

15. Cox PJ, Kirk T, Ashmore T, Willerton K, Evans R, Smith A, et al. Nutritional ketosis alters fuel preference and thereby endurance performance in athletes. Cell Metab. 2016;24(2):256-68.

16. White $H$, Venkatesh $B$. Clinical review: ketones and brain injury. Crit Care (London). 2011;15(2):219.

17. Goossens C, Weckx R, Derde S, Dufour T, Vander Perre S, Pauwels L, et al. Adipose tissue protects against sepsis-induced muscle weakness in mice: from lipolysis to ketones. Crit Care. 2019;23(1):236.

18. Alberti KG, Johnston DG, Gill A, Barnes AJ, Orskov H. Hormonal regulation of ketone-body metabolism in man. Biochem Soc Symp. 1978:43:163-82.

19. Bahnsen M, Burrin JM, Johnston DG, Pernet A, Walker M, Alberti KG. Mechanisms of catecholamine effects on ketogenesis. Am J Phys. 1984; 247(2 Pt 1):E173-80.

20. Beylot M, Picard S, Chambrier C, Vidal H, Laville M, Cohen R, et al. Effect of physiological concentrations of insulin and glucagon on the relationship between nonesterified fatty acids availability and ketone body production in humans. Metabolism. 1991:40(11):1138-46.

21. Keller U, Gerber PP, Stauffacher W. Fatty acid-independent inhibition of hepatic ketone body production by insulin in humans. Am J Phys. 1988; 254(6 Pt 1):E694-9. 
22. Beisel WR, Wannemacher RW Jr. Gluconeogenesis, ureagenesis, and ketogenesis during sepsis. JPEN J Parenter Enteral Nutr. 1980;4(3):277-85.

23. Birkhahn RH, Long CL, Fitkin DL, Busnardo AC, Geiger JW, Blakemore WS. A comparison of the effects of skeletal trauma and surgery on the ketosis of starvation in man. J Trauma. 1981;21(7):513-9.

24. Ohtoshi M, Jikko A, Asano M, Uchida K, Ozawa K, Tobe T. Ketogenesis during sepsis in relation to hepatic energy metabolism. Res Exp Med (Berl). 1984;184(4):209-19.

25. Beylot M, Guiraud M, Grau G, Bouletreau P. Regulation of ketone body flux in septic patients. Am J Phys. 1989;257(5 Pt 1):E665-74.

26. Thiessen SE, Derde S, Derese I, Dufour T, Vega CA, Langouche L, et al. Role of glucagon in catabolism and muscle wasting of critical illness and modulation by nutrition. Am J Respir Crit Care Med. 2017;196(9):1131-43.

27. Fivez T, Kerklaan D, Verbruggen S, Vanhorebeek I, Verstraete S, Tibboel D, et al. Impact of withholding early parenteral nutrition completing enteral nutrition in pediatric critically ill patients (PEPaNIC trial): study protocol for a randomized controlled trial. Trials. 2015;16:202.

28. Boers AMM, Jansen IGH, Brown S, Lingsma HF, Beenen LFM, Devlin TG, et al. Mediation of the relationship between endovascular therapy and functional outcome by follow-up infarct volume in patients with acute ischemic stroke. JAMA Neurol. 2019;76(2):194-202.

29. Guiza F, Vanhorebeek I, Verstraete S, Verlinden I, Derese I, Ingels C, et al. Effect of early parenteral nutrition during paediatric critical illness on DNA methylation as a potential mediator of impaired neurocognitive development: a pre-planned secondary analysis of the PEPaNIC international randomised controlled trial. Lancet Respir Med. 2020;8(3):288-303.

30. Lee H, Herbert RD, Lamb SE, Moseley AM, McAuley JH. Investigating causal mechanisms in randomised controlled trials. Trials. 2019;20(1):524.

31. Williamson DH, Mellanby J, Krebs HA. Enzymic determination of D(-)-beta-hydroxybutyric acid and acetoacetic acid in blood. The Biochem J. 1962:82:90-6.

32. Nomura T, Nomura Y, Tachibana M, Nomura H, Ukai K, Yokoyama R, et al. Alpha 1-adrenergic regulation of ketogenesis in isolated rat hepatocytes. Biochim Biophys Acta. 1991;1092(1):94-100.

33. Jacobs A, Derese I, Vander Perre S, Wouters PJ, Verbruggen S, Billen J, et al. Dynamics and prognostic value of the hypothalamus-pituitary-adrenal axis responses to pediatric critical illness and association with corticosteroid treatment: a prospective observational study. Intensive Care Med. 2020; 46(1):70-81.

34. Hermans G, Wilmer A, Meersseman W, Milants I, Wouters PJ, Bobbaers H, et al. Impact of intensive insulin therapy on neuromuscular complications and ventilator dependency in the medical intensive care unit. Am J Respir Crit Care Med. 2007;175(5):480-9.

35. Van den Berghe G, Schoonheydt K, Becx P, Bruyninckx F, Wouters PJ. Insulin therapy protects the central and peripheral nervous system of intensive care patients. Neurology. 2005;64(8):1348-53.

\section{Publisher's Note}

Springer Nature remains neutral with regard to jurisdictional claims in published maps and institutional affiliations.

Ready to submit your research? Choose BMC and benefit from:

- fast, convenient online submission

- thorough peer review by experienced researchers in your field

- rapid publication on acceptance

- support for research data, including large and complex data types

- gold Open Access which fosters wider collaboration and increased citations

- maximum visibility for your research: over $100 \mathrm{M}$ website views per year

At $\mathrm{BMC}$, research is always in progress.

Learn more biomedcentral.com/submissions 